\title{
Three-dimensional Modeling of Carbon Fiber Cloth Surface from Dense and Scattered Points Cloud
}

\author{
Cheng $\mathrm{Jie}^{*}, 1$ and Chen $\mathrm{Li}^{2}$ \\ ${ }^{1}$ Informatization Center, Tianjin Polytechnic University, Tianjin, 300000, P.R. China \\ ${ }^{2}$ School of Textile, Tianjin Polytechnic University, Tianjin, 300000, P.R. China
}

\begin{abstract}
Fabric surface analysis, as part of fabric analysis, is very important for the textile manufacturing process and is traditionally based on human-labor or image processing which is a conventional automatic method. However, image quality is influenced by ambient light, background light and optical properties of the surface. In this paper, we present a three dimensional modeling techniques for the reconstruction of carbon-fiber fabric surface. Firstly, a dense and scattered points cloud is collected using 3D laser scanning system after necessary data quality analysis. Secondly, the original points cloud is preprocessed according to classification. Finally, three-dimensional fabric surface model is reconstructed using screened Poisson reconstruction algorithm. The experimental results show that the reconstructed model is acceptable.
\end{abstract}

Keywords: 3D laser scanning, 3D reconstruction, Carbon fabric, Detection of outliers, Filtering and denoising.

\section{INTRODUCTION}

Carbon-fiber cloth has received much attention in recent years due to its superior physical and mechanical properties, including low expansion coefficient, high-strength and highmodulus. It is widely used in industry. However, fabric surface analysis has been traditionally depending on human labor, which was time-consuming and lab-intensive. Since 1980, image processing technique has been considered a conventional automatic analysis method. In this method, texture and structural parameters are identified according to gray distribution periods on fabric surface. It means that the quality of images will greatly be influenced by ambient light, background light and optical properties of fabric surface.

3D techniques analyze the collection using 3D coordinates of surface points with the aid of 3D collecting system and algorithms which decrease the effect of light condition to some extent. In textile industry, 3D techniques have been applied into detection and visualization of $3 \mathrm{D}$ model of fabric surface. Photometric stereo is a popular method in micro-analysis of fabric surface. Yang [1] reconstructed the fabric surface using photometric stereo and shape from shadow method. Liu [2] calculated the height of fabric surface from the gradient vector space. Kang [3] reconstructed pilling fabric surface using binary CCD camera and projected stereo vision algorithm and finished pilling rating from $2.5 \mathrm{D}$ depth map. Recently, dense points cloud collected using laser scanning system has been applied into the modeling and analysis of the body [4].

*Address correspondence to this author at the Informatization Center, Tianjin Polytechnic University, Tianjin, 300000, P.R. China;

Tel: +86 13821294571; E-mail: chengjie@tjpu.edu.cn
Laser scanning technique has been used in many fields, including protection of historical relics, sculpture, reverse engineering etc. It can rely little on light condition, collect dense points cloud in short time and receive precise 3D coordinates. What's more, sampling process is simple and convenient. As an efficient and accurate non-contact measuring method, it has been applied in many fields, including protection of historical relics, sculpture, reverse engineering, etc.

In this paper, a dense points cloud of carbon-fiber fabric surface is collected using 3D laser scanning system. According to the distribution of the original points cloud, they are classified and pre-treated in different ways. Finally, fabric surface model is reconstructed from ideal points cloud.

\section{COLLECTION OF 3D POINTS CLOUD OF CARBON-FIBER FABRIC SURFACE}

The collection device is composed of line structured-light sensor (Scan Shark V4ix) and flexible coordinate measuring machine (ROMER) which is produced by Hexagon, as shown in Fig. (1). This device scans 23,000 points per second. The scanning accuracy is $24 \mathrm{um}$.

The sampled fabrics are plain and twill $12 \mathrm{~K}$ carbon fiber fabric, as shown in Fig. (2). Considering the special optical properties of carbon fibers that are all-black and highly reflective, low-illuminate ring lamp is used in order to increase the number of points in per unit area. The scanning distance is about $5 \mathrm{CM}$.

In order to reduce data, it is necessary to select a certain scope of scanning according to the goal of experiment. In practice, at least $2 \times 2$ complete repeats of patterns are needed 
to measure and analyze structural parameters of fabric. Because the cycle numbers of sampled plain and twill carbon fabric are 2 and 8 respectively, the scanning area should not be less than $10 \mathrm{~mm} \times 10 \mathrm{~mm}$ and $54 \mathrm{~mm} \times 54 \mathrm{~mm}$ respectively. If the fabric density measurement which needs to count the number of yarns in warp and weft direction respectively within $10 \mathrm{~cm}$ is taken into account, the scanning area should be added to overcome the lack of points on the edge of scope. Therefore, in collection process, a red laser line whose frequency is $30 \mathrm{HZ}$ is used to scan the square area within $10 \mathrm{~cm} \times 10 \mathrm{~cm}$ and $15 \mathrm{~cm} \times 15 \mathrm{~cm}$ respectively on one side of the plain and twill fabric repeatedly. In order to avoid the deformation on the edge of fabric caused by cutting or stretching, the middle area of large fabric is selected. In the scanning process, the fabric should be plain and tightly stick to the experiment table.

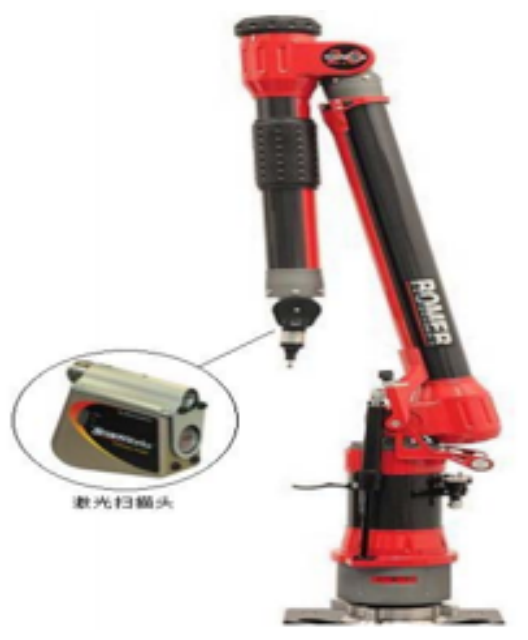

Fig. (1). 3D laser scanning system.

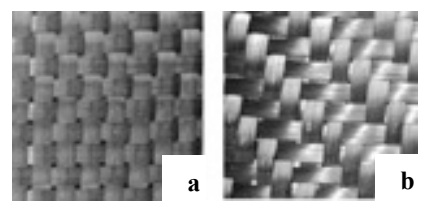

Fig. (2). The sampled fabrics (a) twill (b) plain.

In the experiment, $1,432,846$ original point data of plain fabric and 1,919,669 original points data of twill fabric are collected. The rendered visualization of the two scanning points is illustrated as Fig. (3).

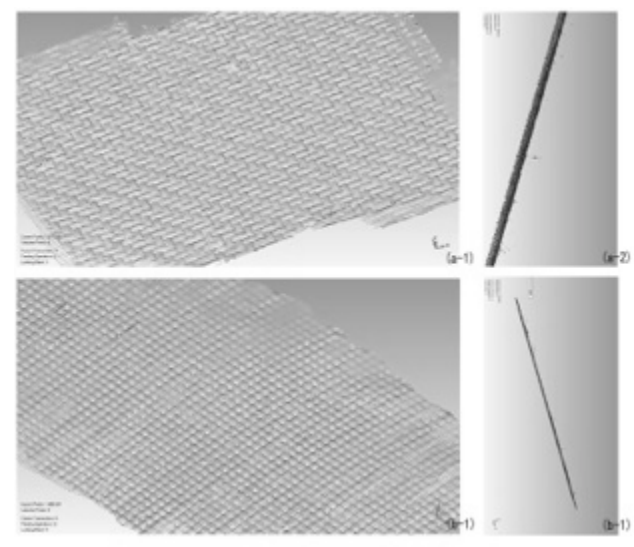

Fig. (3). The rendered visualization of the original scanning points. (a) twill (b) plain.
Once the scanning system has been set up and original points cloud is acquired, 3D model of carbon fiber fabric surface can be constructed according to the following flowchart which is shown in Fig. (4).

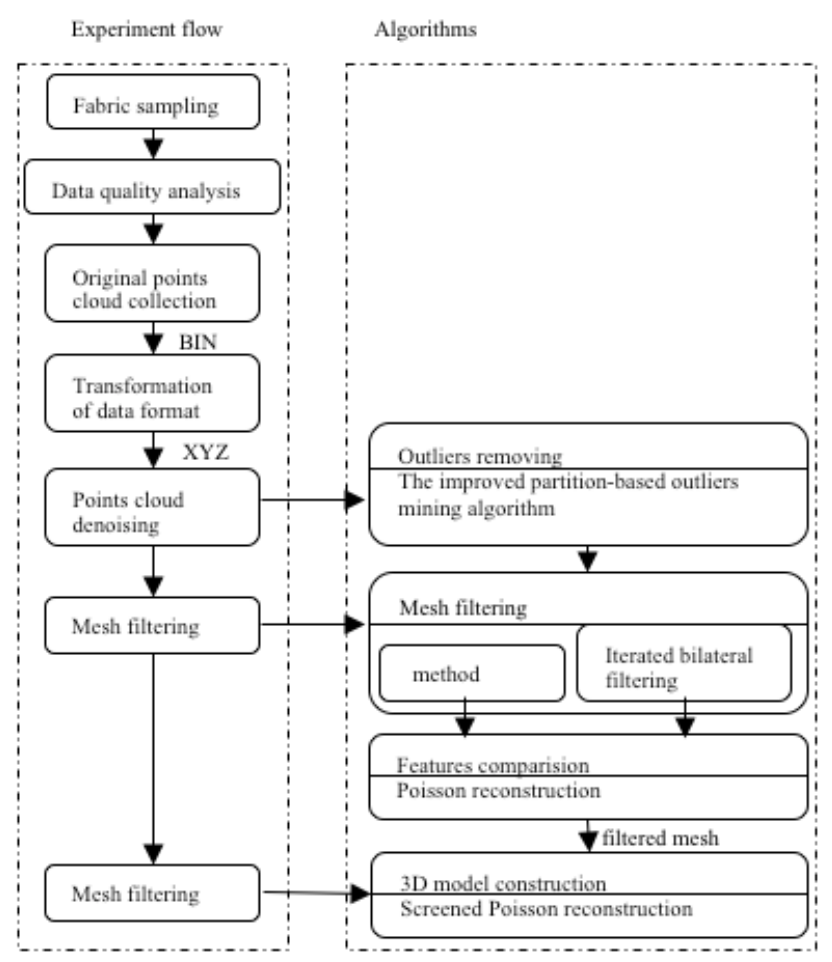

Fig. (4). Flowchart of the provided method of fabric density.

\section{DENOISING OF FABRIC SURFACE}

In Fig. (3), the texture of yarns on two fabrics can be distinguished by eyes clearly. However, the features on most area of fabric surface are not recognized due to holes and noises. Therefore, it is difficult to reconstruct the surface model directly from the original points. These points are classified into outliers and inliers according to distribution and form reasons. Then they are pre-treated in different ways.

\subsection{Outliers Removing}

Outliers are far away from the whole points cloud, and are useless to describe the surface features. They should be removed.

This paper improves Ramaswamy's partition-based outliers mining algorithm [5] that is based on the distance of a point from its $k$ th nearest neighbor. In partition-based algorithm, for a $k$ and point $p, D^{k}(p)$ is denoted as the Euclidean distance of $k$ th the nearest neighbor of $p$. The top $n$ points with the maximum $D^{k}(p)$ are considered as outliers. In the original algorithm, $n$ should be given at first. The improved algorithm uses the average Euclidean distance of $k$ th the nearest neighbor of a point as the metric and removes outliers automatically. 

below.

Main procedures of the improved algorithm are shown as

Firstly, giving the number of points in the $k$ th nearest neighbor which is denoted as $K$. Secondly, computing the Euclidean distance of $k$ th the nearest neighborhood of a point $p$ which is denoted as $D^{k}(p)$ according to

$D^{k}(p)=\sqrt{\sum_{k=1}^{m}\left(x_{i k}-x_{j k}\right)^{2}}$,

where $k=1,2 \ldots K$. Thirdly, sorting these $D^{k}(p)$ s and computing the average Euclidean distance $\bar{d}$. Then, comparing $D^{k}(p) \mathrm{s}$ with $4 \bar{d}$, and marking all points using a flag $F \operatorname{lag}(p)$. If $D^{k}(p)>4 \bar{d}$, the point is an outlier and $\operatorname{Flag}(p)=1$. If $D^{k}(p)<4 \bar{d}$, the point is not an outlier and Flag $(p)=0$. Finally, remove those points whose flag equals one. The diagram of the improved algorithm is shown as Fig. (5).

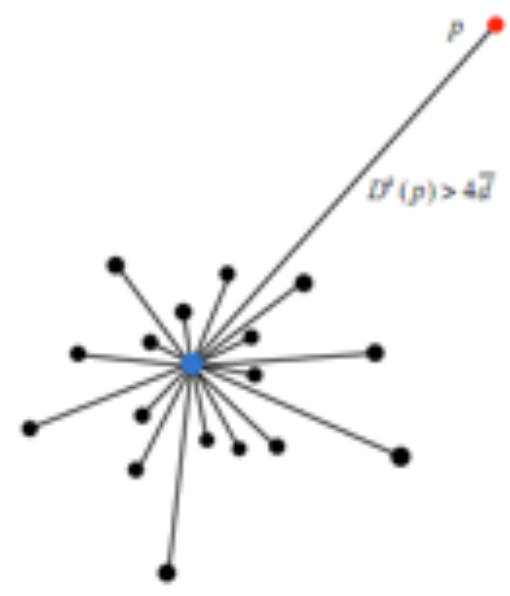

Fig. (5). The diagram of the improved partition-based outliers mining algorithm.

In removing outliers experiment, setting $k=50$. 25,124 outliers on plain surface are detected and removed, which are $1.75 \%$ of the original points. 39,200 outliers on twill surface are detected and removed, which are $2.04 \%$ of the original points.

Then the scattered points cloud is transformed into PLY mesh for filtering in Geomagic studio. There are 617,894 vertices and 1,229,203 triangle patches in plain mesh and 452,550 vertices and 903,065 triangle patches in twill mesh.

\subsection{Mesh Filtering}

Inliers are mixed together with the real point data. They are within the allowance of measurement errors and useful for describing features of fabric surface after necessary preprocessed. During mesh filtering process, inliers are adjusted back to fabric surface.

Assuming a discrete surface signals is a function $x=\left\{x_{1}, \ldots x_{n}\right\}^{t}$ defined on vertices of a polyhedral surface. Laplacian operator of the discrete surface signals is denoted as Equation (1) using the weighted averages over the neighborhoods.
$\Delta x_{i}=\sum_{j \in i^{*}} w_{i j}\left(x_{j}-x_{i}\right)$

where the weights $w_{i j}$ are positive numbers that add up to one. That is $\sum_{j \in i^{*}} w_{i j}=1$.

Because Laplacian operator $x \rightarrow \Delta x$ takes on linear in the signal space of graphics $G$ and it can be operated on $x$ coordinate, it is enough to consider one dimensional graphics. Assuming the weights of edges are decided by the cost of edges that is selected at first time which is denoted as $c_{i j}=c_{j i} \geq 0$, there is

$w_{i j}=c_{i j} / c_{i},\left(c_{i}=\sum_{j \in i^{*}} c_{i j}>0\right)$.

The matrix of costs and the matrix of weights of edge are denoted as $C=\left(c_{i j}\right), W=\left(w_{i j}\right)$. When $j$ is not a neighbor of $i$, the matrix $K$ is defined as $K=I-W$, where $I$ is the same matrix. Laplacian operator that is applied on graphics signals may be denoted as $\Delta x=-K x$.

where $K$ is a symmetric circulant matrix.

$K=\frac{1}{2}\left(\begin{array}{ccccc}2 & -1 & & & -1 \\ -1 & 2 & -1 & & \\ & \cdots & \ldots & \ldots & \\ & & -1 & 2 & -1 \\ -1 & & & -1 & 2\end{array}\right)$.

Gaussian smoothing and scale space theory are common methods among smoothing algorithms based on convolution for parameters curve. Using these two methods, Laplacian smoothing algorithm can be denoted as Equation (2) using matrix.

$x=x+\lambda \Delta x=(1-\lambda k) x=f(K) x$

In Equation (2), scale factor $\lambda(0<\lambda<1)$ is used to control diffusion rate. $f(K)$ is acquired by evaluating the polynomial transfer function $f(k)=(1-\lambda k)$ in matrix $K$. For $N$ times iteration, there is $f(k)=(1-\lambda k)^{N}$, where $f(k)$ is the transfer function of filter.

The above method is regarded as the classical Laplacian smoothing algorithm [6]. The main idea is to correct the location of a vertex by calculating the average of the locations of vertices in the neighborhood on the mesh [7]. Therefore, the correction process will lead to obvious deformation and shrinkage. To solve this problem, Taubin [8], Vollmer [7] and Desbrun [9] improved the Laplacian algorithm. Among these improved methods, Taubin's $\lambda / \mu$ method is based on a transfer function of the filter as shown in Equation (3). It performs the Gaussian smoothing step of Equation (3) with positive scale factor $\lambda$ for the shrinking step and negative scale factor $\mu$ for un-shrinking step. In this way, high frequencies diffuse in different directions 
unevenly. Then high frequencies are removed and low frequencies are retained and strengthened.

$f(k)=(1-\lambda k)(1-\mu k)^{N / 2}$

where $\lambda>0, \mu<-\lambda<0, N$ is an even number.

Laplacian smoothing algorithm and its improved algorithms are all isotropic filtering methods, in which the filter acts independently of direction. It is difficult for these filters to preserve prominent directional mesh features. Therefore, anisotropic filtering schemes are proposed. In such approaches, anisotropic diffusion equations are built. The diffusion factors which depend on main curvature and main direction of curvatures are sensitive to obvious characteristics on surface. And diffusion curvature on certain direction of sharp edge and corners can be decreased [10]. Fleishman's iterated bilateral filter [11] is a typical anisotropic method. This algorithm defines a local parameter space for every vertex using the tangent plane to the mesh at a vertex. The vertex is adjusted along the normal direction $d$ of the plane. $d$ is defined as Equation (4).

$d=\frac{\left.\left.\sum_{\mathrm{q}_{i} \in N b(\mathrm{v})} W_{c}\left(\| \mathrm{v}-\mathrm{q}_{\mathrm{i}}||\right) W_{s}\right\rangle\left|\left\langle\mathrm{n}, \mathrm{v}-\mathrm{q}_{\mathrm{i}}\right\rangle\right| \mid\right)\left\langle\mathrm{n}, \mathrm{v}-\mathrm{q}_{\mathrm{i}}\right\rangle}{\sum_{\mathrm{q}_{i} \in N b(\mathrm{v})} W_{c}\left(\| \mathrm{v}-\mathrm{q}_{\mathrm{i}}||\right) W_{s}\left(\|\left\langle\mathbf{n}, \mathrm{v}-\mathrm{q}_{\mathrm{i}}\right\rangle \mid\right)}$

where $\left\{\mathbf{q}_{\mathbf{i}}\right\}$ denotes the neighborhood of $\mathbf{v} .|| \mathbf{v}-\mathbf{q}_{\mathbf{i}} \|$ denotes the similarity of vertices. $\left\langle\mathrm{n}, \mathrm{v}-\mathrm{q}_{\mathrm{i}}\right\rangle$ denotes the similarity of height.

In filtering experiment, Taubin's $\lambda / \mu$ method and iterated bilateral filtering algorithm [11] are performed on the mesh of plain and twill surface. Considering that the two filtering methods only update the positions of vertices while not delete or add the vertices on mesh, this paper performs 3D reconstruction of fabric surface model using Poisson reconstruction [12], and compares the amount of vertices and triangle patches on meshes which are filtered using Taubin's $\lambda / \mu$ method and Fleishman's bilateral filtering algorithm respectively. The mesh which includes more vertices and triangle patches tends to have more features and is fitful to be the input mesh for reconstruction. The amount of vertices and triangle patches are compared, as shown in Table $\mathbf{1 .}$

The experimental results show that there are more vertices and triangle patches in $\lambda / \mu$ filtered mesh of both fabrics than in mesh which removed outliers and iterative bilateral filtered mesh. It concludes that $\lambda / u$ algorithm tends to preserve and strengthen more features and is appropriate to filter the fabric mesh whose texture is only several micrometers high. When $\lambda=0.3, \mu=-0.43$ and iterating 9 times, meshes are filtered well and preserve more features. The rendered visualizations of filtered mesh are shown in Fig. (6).
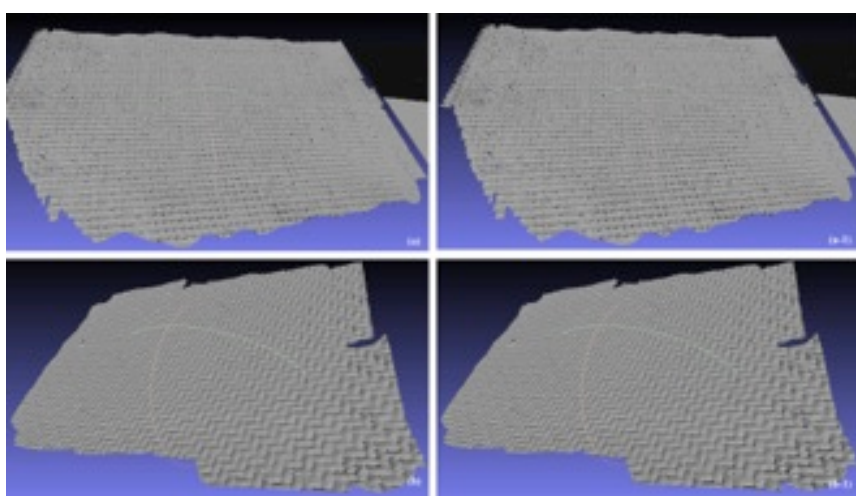

Fig. (6). The rendered visualization $\lambda / \mu$ filtered mesh. (a) original plain mesh (a-1) filtered plain mesh (b) original plain mesh (b-1) filtered plain mesh.

Therefore, $\lambda / \mu$ filtered mesh is taken as the input mesh of next reconstruction.

\section{3D RECONSTRUCTION OF FABRIC SURFACE}

Although outliers are removed and features on fabric surface are stretchered, there are still lots of holes and noises in pre-treated points cloud. It is necessary to further constrain point data, recover the topology structure of $3 \mathrm{D}$ model and reconstruct the geometry model of fabric surface in $3 \mathrm{D}$ space.

Poisson reconstruction [12] is an implicit fitting method. It is global solution that considers all the data at once, without resorting to heuristic partitioning or blending. Thus, it robustly approximates noisy data. Meanwhile, it admits a hierarchy of locally supported functions, and its solution reduces to a well-conditioned sparse linear system.

The key insight of Poisson reconstruction [12] method is that the gradient of the indicator function is a vector field that equals zero almost everywhere, except at points near the surface, where it is equal to the inward surface normal. Because the indicator function is a piecewise constant function, explicit computation of its gradient field would result in a vector field with unbounded values at the surface boundary. To avoid this, we convolve the indicator function with a smoothing filter and consider the gradient field of the

Table 1. The comparison of the amount of vertices and triangle patches on two filtered meshes.

\begin{tabular}{|c|c|c|c|c|}
\hline \multirow{2}{*}{} & \multicolumn{2}{|c|}{ Plain } & \multicolumn{2}{c|}{ Twill } \\
\cline { 2 - 5 } & Vertices & Triangle Patches & Vertices & 762,249 \\
\hline \hline Mesh which is removed outliers & $1,243,623$ & $2,487,047$ & 767,205 & $1,524,334$ \\
\hline$\lambda / \mu$ filtered mesh & $1,260,725$ & $2,521,237$ & $7,534,246$ \\
\hline Iterative bilateral filtered mesh & $1,209,406$ & $2,418,617$ & 7,965 & $1,503,766$ \\
\hline
\end{tabular}


smoothed function. Then, the gradient of smoothed indicator function is equal to the vector field obtained by smoothing the surface normal field, which is shown in Equation (4).

$\nabla\left(X_{M} * \tilde{F}\right)\left(q_{0}\right)=\int_{\partial M} \tilde{F}_{p}\left(q_{0}\right) \vec{N}_{\partial M}(p) d p$

where $X_{M}$ is the edge of the 3D object, $\tilde{F}(q)$ is a smoothing filter, $\vec{N}_{\partial M}(p)$ is the inward normal surface of point $P$ ( $P \in \partial M$ ). If the curve of the surface $\partial M$ is divided into several distinct patches $P_{s}\left(P_{s} \subset \partial M\right)$ using the input point set $S$, the surface normal field integral will be approximated as the discrete summation of sampled points, as shown is Equation (5).

$\nabla\left(X_{M} * \tilde{F}\right)(q)=\sum_{s \in S} \int_{P_{s}} \tilde{F}_{p}(q) \vec{N}_{\partial M}(p) d p \approx \sum_{s \in S}\left|P_{s}\right| \tilde{F}_{s . p}(q) s \vec{N} \equiv \vec{V}(q)$

Then surface fitting can be realized by approximating the indicator function of the model and extracting iso-surface. The variational problem transforms into finding a scalar function $X$ whose gradient best approximates a vector field $\tilde{V}$ defined by the samples. That is to compute the minimum of Equation (6).

$E(X)=\int\|\nabla X(p)-\vec{V}(p)\|^{2} d p$

However, $\vec{V}$ is generally not integrable. To find the best least-squares approximate solution of Equation (6), the divergence of gradient (Laplacian of the scalar function $X$ ) is taken place to the gradient using Euler-Lagrange equation. Then, computing the scalar function $X$ whose divergence of gradient equals the divergence of the vector field, as shown in equation (7).

$\Delta X \equiv \nabla \cdot \nabla X=\nabla \cdot \vec{V}$

where $\Delta$ is the Laplacian operator. This is a standard Poisson problem.

The deficiency of Poisson reconstruction method lies that it corrects indicator function using a single global offset. However, it is difficult to select an appropriate global offset. To solve this problem, Kazhdan [13] proposes to explicitly interpolate point constraints. The approach adds a term to penalize the function's deviation from zero at the samples to the energy of Equation (8).

$E(X)=\int\|\vec{V}(p)-\nabla X(p)\|^{2} d p+\frac{\alpha \cdot \operatorname{Area}(S)}{\sum_{p \in P} w(p)} \sum_{p \in P} w(p) X^{2}$

where $\alpha$ is a weight to trade off the importance of fitting the gradients and fitting values, and $\operatorname{Area}(S)$ is the area of the reconstructed surface that is estimated by computing the local sampling density. Here, set $w(p)=1$. The energy Equation (8) can be expressed concisely as:

$E(X)=\langle\vec{V}-\nabla X, \vec{V}-\nabla X\rangle_{[0,1]^{3}}+\alpha\langle X, X\rangle_{(w, P)}$

where $\langle\cdot,\rangle_{(w, P)}$ is the bilinear, symmetric, positive, semidefinite expression on the space of functions in the unit- cube function space, estimated by taking the weighted sum of function values.

$\langle F, G\rangle_{(w, P)}=\frac{\operatorname{Area}(P)}{\sum_{p \in P} w(p)} \sum_{p \in P} w(p) \cdot F(p) \cdot G(p)$

The energy in Equation (9) combines a gradient constraint integrated over the spatial domain with a value constraint summed at discrete points. The minimum of it can be explained to the screened Poisson equation, expressed by an operator $\tilde{I}$, which is shown in Equation (11).

$(\Delta-a \tilde{I}) X=\nabla \cdot \vec{V}$

Screened Poisson equation can be solved by cascade multi-grid method.

In reconstruction experiments, the input mesh is the $\lambda / \mu$ filtered mesh, in which $\lambda=0.3, \mu=-0.43$ and iterates 9 times. Poisson reconstruction algorithm and screened Poisson reconstruction algorithm are conducted to reconstruct plain and twill surface model at octree depth from 6 to 12. The amounts of vertices and triangle patches at each octree depth are shown in Fig. (7).

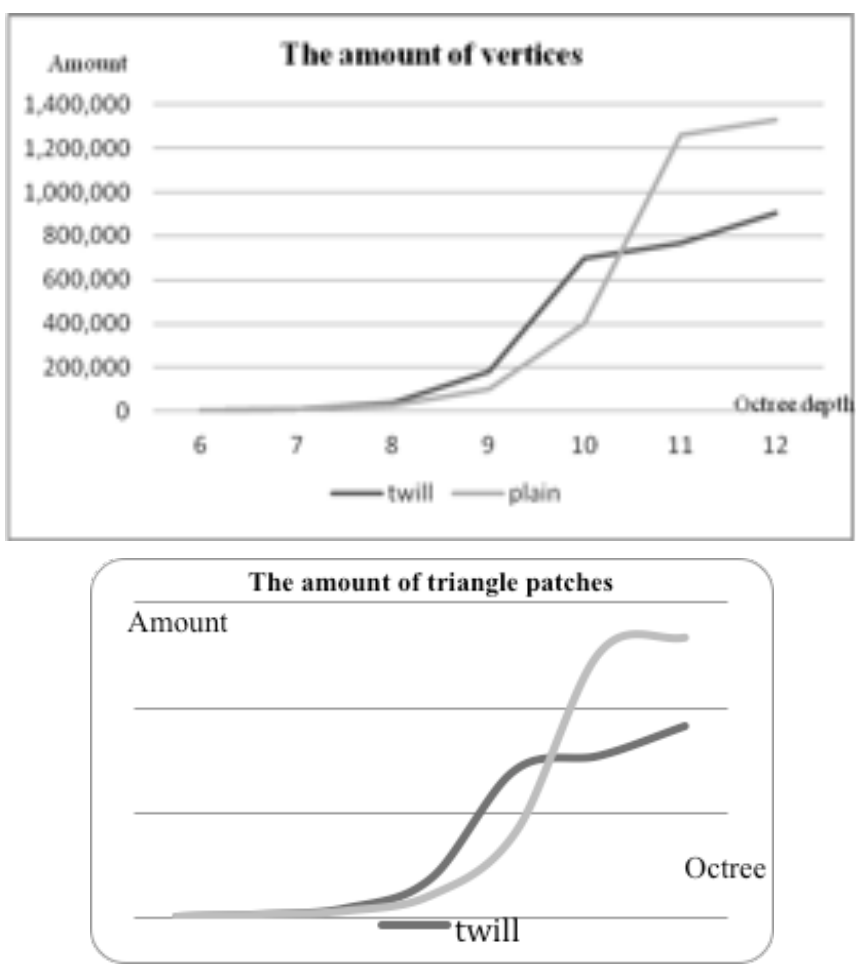

Fig. (7). The amounts of vertices and triangle patches at each octree depth.

The results show that the effects of octree depth 11 and 12 are better than at octree depth 10 . The effects at octree depth 11 and 12 have little difference. When the octree depth adds one, the execution time will add $50-100 \%$, as shown in Fig. (9). In order to acquire better effects and efficiency, the requirement of analysis is satisfied when the depth of octree is set to 11. It is unnecessary to reduce efficiency to reconstruct finer features. The rendered visualization of reconstructed 3D model shows in Fig. (8), where octree depth is 11. 

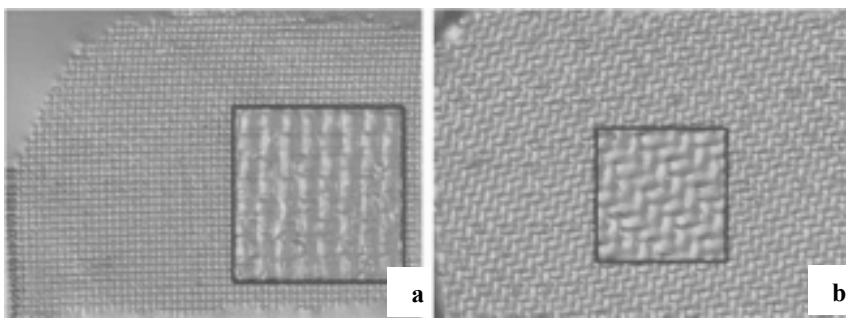

Fig. (8). The rendered visualization of the reconstructed 3D model of the plain and twill surface at octree depths 11. (a) twill (b) plain.

running time at each octree depth

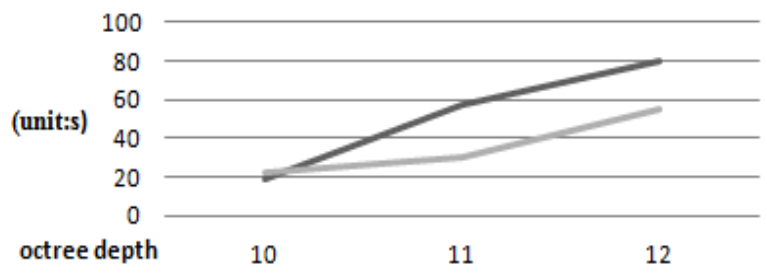

3D model of plain $\quad 3 \mathrm{D}$ model of twill

Fig. (9). Running time of screened Poisson reconstruction at octree depths 10,11 and 12 .

Because the texture of carbon fabric is subtle and allblack, it is not objective and scientific to analyze features of texture and evaluate the effect of reconstruction throw eyes. Consequently, this paper tests the effects through the following method. Contrast the width of fabric yarn which is measured with vernier caliper with the average width which is picked up in Geomagic of five times. Assume the measurement error of model is $\delta=\left|x_{\text {Geomagic }}-x_{\text {fabric }}\right|$. The results of measurement of fabric and model are compared which is shown in Table 2.

Table 2. The comparison between the measurement of fabric and model (Unit: $\mathbf{m m}$ ).

\begin{tabular}{|c|c|c|c|c|}
\hline & & $\begin{array}{c}\text { Measurement } \\
\text { of Fabric }\end{array}$ & $\begin{array}{c}\text { Measurement } \\
\text { of Model }\end{array}$ & Error \\
\hline \hline \multirow{2}{*}{ Plain } & Weft & 3.02 & 2.896 & 0.124 \\
\cline { 2 - 5 } & Warp & 3.04 & 2.911 & 0.129 \\
\hline \multirow{2}{*}{ Twill } & Weft & 3.03 & 2.903 & 0.127 \\
\cline { 2 - 5 } & Warp & 3.04 & 2.947 & 0.093 \\
\hline
\end{tabular}

The experimental results show that the average measurement error of the width of warp is $0.11 \mathrm{~mm}$ and the average measurement error of the width of weft is $0.13 \mathrm{~mm}$. Here, the accuracy of result is decided by the accuracy of scanning, reconstruction and picking up.

\section{CONCLUSION}

In this paper, original points cloud of fabric surface is collected with the aid of 3D laser scanning system which is composed of line structured-light sensor and flexible coordinate measuring machine. $1.895 \%$ outliers are removed using the partition-based outliers mining algorithm. Then the fabric surface mesh is smoothed using Taubin's $\lambda / \mu$ method. When $\lambda=0.3, \mu=-0.43$ and iterate 9 times, meshes are well filtered and preserved more features. Finally the fabric surface models are reconstructed using screened poisson reconstruction. When the depth of octree equals 11 , features on the reconstructed $3 \mathrm{D}$ models and running efficiency are satisfactory.

\section{CONFLICT OF INTEREST}

The authors confirm that this article content has no conflict of interest.

\section{ACKNOWLEDGEMENTS}

Declared none.

\section{REFERENCES}

[1] X B Yang. Computer vision on objective assessment of fabric wrinkle grade. PHD thsis, Jiangnan university, Wuxi, China, 2003.

[2] R X Liu, Study on objective assessment system of fabric pleat grade. PHD thsis, Jiangnan university, Wuxi, China, 2012.

[3] T J Kang, D H Cho, S M Kim. Objective evaluation of fabric pilling using stereovision. Textile Rese J 2004; 74(11): 1013-1017.

[4] H Q Huang, B X MO, Guo Qi-lian, et al. Garment patterns generating based on 3D body scanning. J Textile Rese 2010; $9(31)$ : $132-142$.

[5] S Ramaswamy, R Rastogi, K Shim. Efficient algorithms for mining outliers from large data sets. ACM SIGMOD Record ACM 2003; 29(2): 427-438.

[6] Field D A. Laplacian smoothing and Delaunay triangulations. Comm Appl Numer Meth 1988; 4(6):709-712.

[7] J Vollmer, R Mencl, H Mueller. Improved Laplacian smoothing of noisy surface meshes. Comput Graph Forum Blackwell Pub Ltd, 1999; 18(3): 131-8.

[8] G Taubin. A signal processing approach to fair surface design". Proceedings of the $22^{\text {nd }}$ annual conference on Computer graphics and interactive techniques, ACM, 1995, pp. 351-358.

[9] M Desbrun, M Meyer, P Sehroder et al. Implicit fairing of irregular meshes using diffusion and curvature flow. Comp Graph 1999; pp. 317-24.

[10] X Sun, P L Rosin, R R Martin, et al. Fast and effective featurepreserving mesh denoising, Visualiz Comput Graph, 2007; 13(5): 925-38.

[11] S Fleishman, I Drori, D Cohen-Or. Bilateral mesh denoising. ACM Transact Graph 2003; 22(3): 950-3.

[12] M Kazhdan, $M$ Bolitho, and $H$ Hoppe. Poisson surface reconstruction. eurographics symposium on geometry processing 2006.

[13] M Kazhdan, H Hoppe. Screened poisson surface reconstruction ACM Transact Graph 2013; 32(3): 29. 